\title{
LETTER
}

\section{Determining the optimal number of lung ultrasound zones to monitor COVID-19 patients: can we keep it ultra-short and ultra-simple?}

\author{
Micah L. A. Heldeweg ${ }^{1,2^{*}} \mathbb{0}$, Arthur W. E. Lieveld ${ }^{1,2,3}$, Harm J. de Grooth ${ }^{1}$, Leo M. A. Heunks ${ }^{1}$ \\ and Pieter R. Tuinman ${ }^{1,2}$ on behalf of the ALIFE study group
}

(c) 2021 Springer-Verlag GmbH Germany, part of Springer Nature

With much interest, we read the correspondence from Mento and colleagues, as well as the original study by Volpicelli and colleagues $[1,2]$. We would like to contribute original study results to move the discourse on optimal lung ultrasound methodology forward.

Although the work by Mento provides an interesting perspective, we believe that the method used to study agreement between lung ultrasound protocols may have inherently led to the presented conclusions.

The authors compare the proportion of worst lung ultrasound scores (LUS) across different protocols with a subjectively selected 14-zone protocol as reference standard.

First, whether the reference standard accurately represents total pulmonary involvement is uncertain. In fact, previous research has shown equivalence of both 6- or 12-zone protocols compared to gold standard chest computed tomography (CT) [3, 4]. Second, the 14-zone protocol's overrepresentation of posterior zones (43\%) constitutes a scan-location bias, which is problematic when examining disease with gravity-dependent distribution. Consequently, comparing worst scores of predominantly posterior LUS protocol to worst scores of predominantly lateral or anterior LUS protocols inevitably leads to lower agreement. Third, exclusively evaluating worst LUS disregards a plethora of particulars needed to assess true agreement between protocols.

We present the results of a study with robust methods to comprehensively evaluate agreement between LUS protocols.

We performed a prospective observational study at the tertiary intensive care unit of the Amsterdam University Medical Centers, location VUmc. The study was approved by the local ethics board and need for informed consent was waived. A total of 191 examinations from 102 critically ill patients $(81.4 \%$ male; mean age $64.9 \pm 11.4)$ affected by coronavirus disease 2019 (COVID-19) were examined and analyzed. Full methodology is described in Supplementary S1. Reference test was a 12-zone LUS protocol which has shown to have monitoring equivalence to $\mathrm{CT}$ and index test was a 6-zone LUS protocol (Fig. 1A) [4]. Each LUS zone was scored from 0 (A-pattern) to 3 (consolidation). A LUS index $($ LUSI $=($ LUS/LUS achievable $) \times 100)$ was calculated for both.

Agreement was tested using Spearman's correlation coefficient, Bland-Altman plot, and smallest

\footnotetext{
*Correspondence: M.heldeweg@amsterdamumc.n

${ }^{1}$ Department of Intensive Care Medicine, Amsterdam University Medical Centers, Location VUmc, Postbox 7507, 1081HV Amsterdam, The Netherlands

Full author information is available at the end of the article
} 

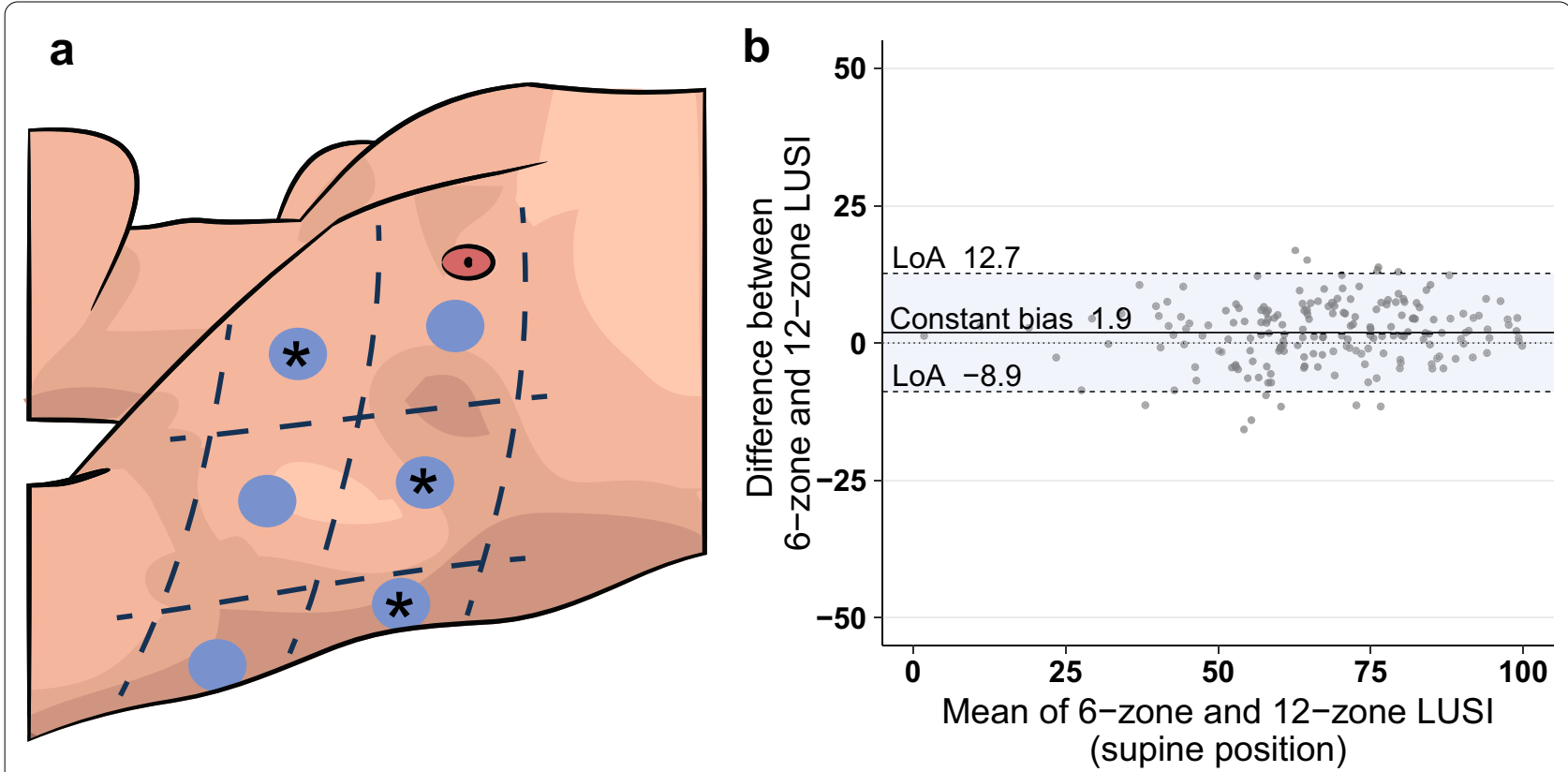

Fig. 1 Lung ultrasound reference standard (blue) and index test (asterix) (a), and the Bland-Altman plot (b). Each point represents agreement between the index and reference test in one examination in one patient. A jitter effect was added to improve visualization of data and avoid direct overlap of multiple examinations. LOA Limits of Agreement, LUS/ lung ultrasound score index-the lung ultrasound score expressed as a percentage of total score achievable

detectable change with accompanying 95\% confidence intervals (Supplementary S2).

The Spearman's correlation coefficient was 0.944, indicating a strong correlation. The Bland-Altman plot (Fig. 1B) exhibited a constant bias, indicating that 6-zone LUS was consistently $1.9 \%$ (95\% CI $1.1 \%$, 2.7\%) higher than 12-zone LUS. No proportional bias was found, signifying that imaging protocols agreed equally across disease severities. The limits of agreement of $10.8 \%$ (95\% CI $7.4 \%, 14.2 \%$ ) were smaller than the calculated smallest detectable change of $17.4 \%$ (95\% CI $11.8 \%, 26.1 \%)$ ( $p=0.019$, derived from 10,000 bootstrapped comparisons), indicating that differences between protocols were smaller than the measurement error (comparing each protocol to itself would have led to similar limits of agreement).

Monitoring COVID-19 with more than six zones does not appear to provide additional clinical information. This is important, because much of lung ultrasound's value is owed to its efficient bedside applicability, particularly in time and resource strained settings, such as the COVID-19 pandemic. Although these results need to be validated comprehensively, this study agrees with previous investigations concerning optimal number of lung ultrasound zones: less is more [5].

\section{Supplementary Information}

The online version contains supplementary material available at https://doi. org/10.1007/s00134-021-06463-6.

\section{Author details}

${ }^{1}$ Department of Intensive Care Medicine, Amsterdam University Medical Centers, Location VUmc, Postbox 7507, 1081 HV Amsterdam, The Netherlands. ${ }^{2}$ Amsterdam Leiden IC Focused Echography, Amsterdam, The Netherlands.

${ }^{3}$ Department of Internal Medicine, Amsterdam UMC, Section Acute Medicine, Location VUmc, Amsterdam, The Netherlands.

\section{Acknowledgements}

ALIFE study group: Micah LA Heldeweg, Arthur WE Lieveld, Mark E Haaksma, Jasper M Smit (patient inclusions for this sub-study), Jorge E Lopez Matta, Carlos V Elzo Kraemer, David J van Westerloo, Pieter RTuinman (project leaders)

Funding

No funding was received for this study. 
Availability of data, material, and code

The data sets used and/or analyzed, as well as the custom R code, during the current study are available from the corresponding author on reasonable request.

\section{Declarations}

\section{Conflicts of interest}

The authors declare that they have no competing interests.

\section{Ethics approval and consent to participate}

The local ethics committee ('Medisch-ethische toetsingscommissie') approved this study (Registration ID: 2020.011). Consent for participation was not applicable as ultrasound measurements were carried out as part of routine clinical examination.

\section{Consent for publication}

Consent for publication was waived by the local ethics board.

\section{Publisher's Note}

Springer Nature remains neutral with regard to jurisdictional claims in published maps and institutional affiliations.

Received: 28 May 2021 Accepted: 18 June 2021

Published online: 26 June 2021

\section{References}

1. Mento F, Perrone T, Fiengo A et al (2021) Limiting the areas inspected by lung ultrasound leads to an underestimation of COVID-19 patients' condition. Intensive Care Med 11:1-2. https://doi.org/10.1007/s00134-021-06407-0

2. Volpicelli G, Gargani L, Perlini S et al (2021) Lung ultrasound for the early diagnosis of COVID-19 pneumonia: an international multicenter study. Intensive Care Med 1 47(4):444-454. https://doi.org/10.1007/s00134-021-06373-7

3. Pisani L, Vercesi V, van Tongeren PSI et al (2019) The diagnostic accuracy for ARDS of global versus regional lung ultrasound scores-a post hoc analysis of an observational study in invasively ventilated ICU patients. Intensive Care Med Exp 25 7(S1):44. https://doi.org/10.1186/ s40635-019-0241-6

4. Heldeweg M, Matta J, Haaksma M et al (2020) Lung ultrasound and computed tomography to monitor COVID-19 pneumonia in critically ill patients: a two-center prospective cohort study. Intensive Care Med Exp 25 9(1):1. https://doi.org/10.1186/s40635-020-00367-3

5. Cox EGM, Wiersema R, Wong A et al (2020) Six versus eight and twentyeight scan sites for B-line assessment: differences in examination time and findings. Intensive Care Med 46(5):1063-1064. https://doi.org/10. 1007/s00134-020-06004-7 